\title{
Anesthetic Management of Surgical Repair of Coarctation of Aorta in Neonates and Infants
}

\author{
Nabil A Mageed*, Ibrahim I Abd El Baser and Hani I Taman \\ Department of Anesthesia and Surgical Intensive Care, Mansoura University, Egypt
}

*Corresponding author: Nabil A Mageed, Department of Anesthesia and Surgical Intensive Care, Mansoura University, Egypt.

Received Date: April 08, 2020

Published Date: April 21, 2020

\begin{abstract}
Coarctation of aorta $(\mathrm{CoA})$ is a localized narrowing of descending thoracic aorta most commonly between left subclavian artery and ductus arteriosus causing proximal hypertension and distal hypoperfusion. The clinical presentation of CoA in neonates and infants varies from acute hemodynamic collapse to weak or absent arterial femoral pulsation. Keeping the patency of ductus arteriosus with prostaglandin E1 infusion is lifesaving, once the diagnosis of severe CoA is established. Transthoracic echocardiography is the cornerstone for the diagnosis and follow-up of CoA. Inotropic drugs such as dobutamine, dopamine and epinephrine are indicated to maintain and stabilize the hemodynamics in neonates and infants with acute heart failure. Resection of the constricted segment with end to end anastomosis is the standard surgical approach. Preductal invasive arterial pressure monitoring via an arterial catheter placed in the right arm is essential. During surgery, passive cooling of the patients to a temperature of $35^{\circ} \mathrm{C}$ is recommended in to reduce the risk of neurological injury. Clamping the aorta aggravates the proximal hypertension and cause distal body hypoperfusion with increased risk of spinal cord injury. The aim of this review is to declare the anesthetic management of neonates and infants undergoing surgical correction of CoA and how to reduce the risk of aortic cross clamping.
\end{abstract}

Keywords: Coarctation; Anesthetic management; Surgical repair; Neonates; Infants

\section{Introduction}

Coarctation of the aorta ( $\mathrm{CoA})$ is a congenital narrowing of the descending thoracic segment of aorta, most commonly between the origin of left subclavian artery and the aortic end of ductus arteriosus. In case of severe aortic obstruction, the hemodynamic stability will be dependent only on blood flow through the patent ductus arteriosus, therefore CoA is classified as a critical congenital cardiac anomaly [1]. The incidence of CoA is about $6-8 \%$ of all congenital heart diseases (CHD) and 4 for each 10,000 live births [2]. It is incidence is higher in males than females. CoA is usually associated with other CHD such as bicuspid aortic valve, patent ductus arteriosus, ventricular septal defect, and transposition of great arteries. The most associated cardiac anomaly is bicuspid aortic valve with a co-incidence about $45-62 \%[3,4]$.

\section{Clinical Presentation}

The severity and the timing of clinical presentation of CoA are dependent on the degree of aortic obstruction, associated cardiac anomalies and the development of collateral circulation. The clinical presentation of $\mathrm{CoA}$ in neonates and infants varies from acute hemodynamic collapse to weak or absent arterial femoral pulsation.

\section{Early neonatal collapse}

Regardless the severity of aortic obstruction, neonates are usually asymptomatic at the time of birth because the patent ductus arteriosus (PDA) partially maintains the blood supply to the body distal to aortic obstruction. After spontaneous closure of PDA, neonates with severe CoA develop cardiogenic shock and acute hemodynamic collapse. The clinical findings include, weak or absent femoral pulse, manifestations of low cardiac output as skin mottling and prolonged capillary refill, inappropriate feeding, metabolic acidosis, oliguria and even anuria, and profound hypotension. Keeping the patency of ductus arteriosus with prostaglandin E1 infusion is lifesaving, once the diagnosis of severe CoA is established. During infancy, the occurrence of cardiogenic shock is uncommon presentation but remains a possibility. Pulse oximetry of both upper and lower limbs is of great value for the 
screening neontes with severe coarctation when associated with PDA, as the upper limbs are supplied with well oxygenated blood while the lower limbs receive non oxygenated blood due to right to left blood shunting through PDA [5].

\section{Late presentation}

If the degree of aortic obstruction is not so severe and in the presence of collateral blood supply that perfuse the body distal to CoA, infants can remain without any symptoms for a long period. In these patients, the left ventricle is exposed to a very high afterload and will therefore be working at very high pressure. The high afterload caused by aortic obstruction results in marked hypertrophy of left ventricle and dysfunction with development of pulmonary edema in some infants. Infant's parents may complain from gradually worsening dyspnea and failure to thrive. The clinical findings include slow rising radial pulses, weak or absent femoral pulses, an interscapular systolic murmur. The blood pressure measurement in the four limbs of infants is not reliable test as it is difficult and inaccurate [1].

\section{Diagnostic Imaging}

Transthoracic echocardiography (TTE) is usually used for the diagnosis and follow-up of coarctation of aorta. Fetal echocardiography (FE) can be used for prenatal diagnosis of coarctation. Advanced diagnostic imaging includes Cardiac MRI (cMRI) and cardiac computed tomography (CT). Cardiac catheterization gives us an accurate diagnosis and is primarily indicated for interventional management.

\section{Fetal echocardiography}

Intrauterine detection of CoA allows early counselling, appropriate planning of delivery and avoidance of neonatal cardiac emergencies [6]. Fetal echocardiography may allow the prenatal diagnosis of CoA, but the detection rate remains low as it can view only severe CoA while the fetus is in the proper position for aortic arch imaging [7].

\section{Transthoracic echocardiography}

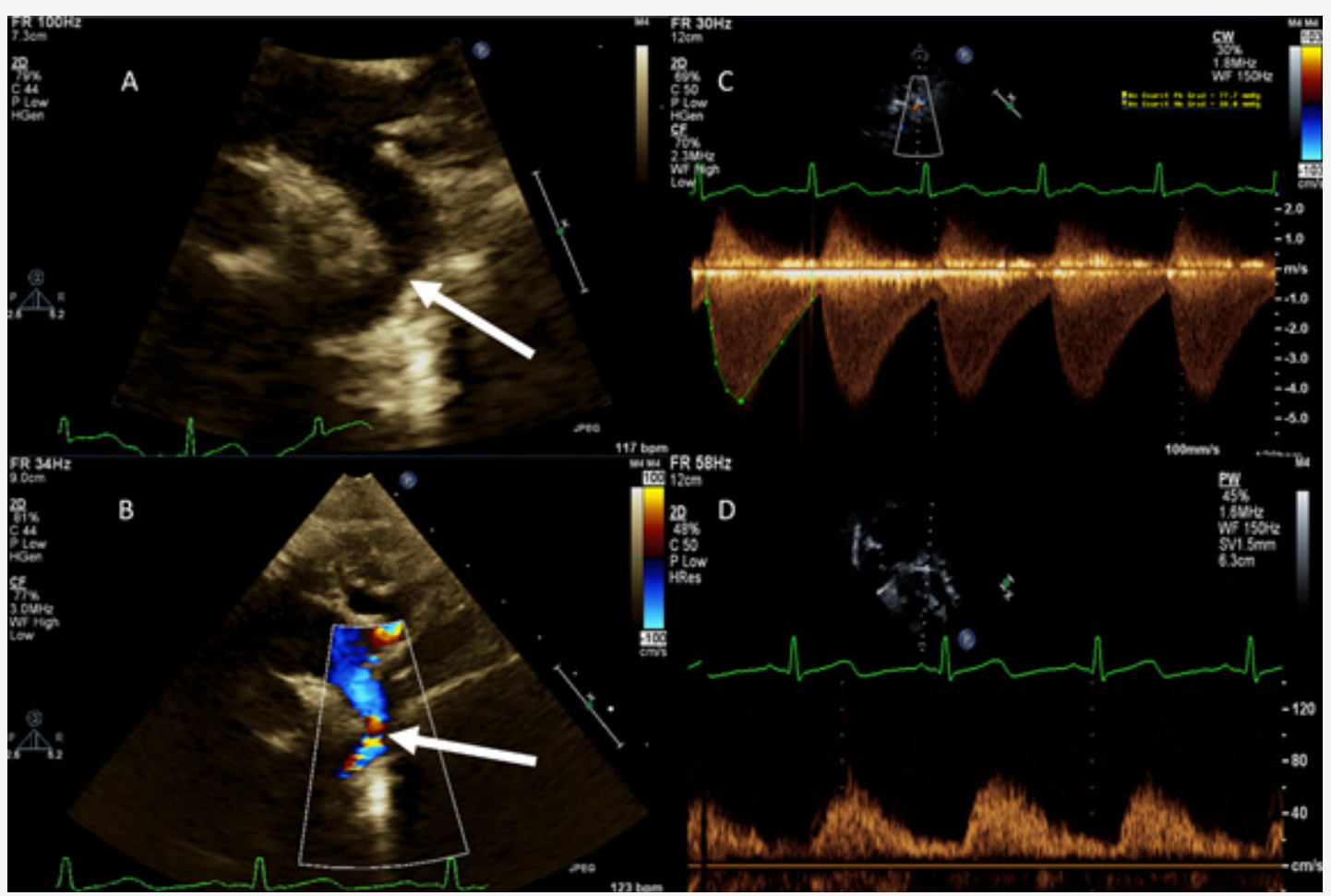

Figure 1: Transthoracic echocardiographic different views of CoA [9].

Transthoracic Echocardiography is the classic imaging technique for the detection and follow-up of CoA in infants and neonates. TTE determines the site of CoA and the severity of constriction, illustrates the anatomy of aortic arch and detects the associated cardiac anomalies [8] (Figure 1).

\section{Transesophageal echocardiography}

Transoesophageal echocardiography is rarely used as a diagnostic tool of $\mathrm{CoA}$ as its invasive imaging technique that requires general anesthesia and the limited imaging views for aortic arch [10].

\section{Computed tomography (CT)}

Three dimensional CT and CT angiography can visualize intra and extra cardiac structures with very high resolution [11]. Computed Tomography imaging produces clear images without disturbing artifacts due to metallic objects. This is of great value for imaging the aortic arch during insertion of aortic stents to bypass the obstruction [12] (Figure 2). 


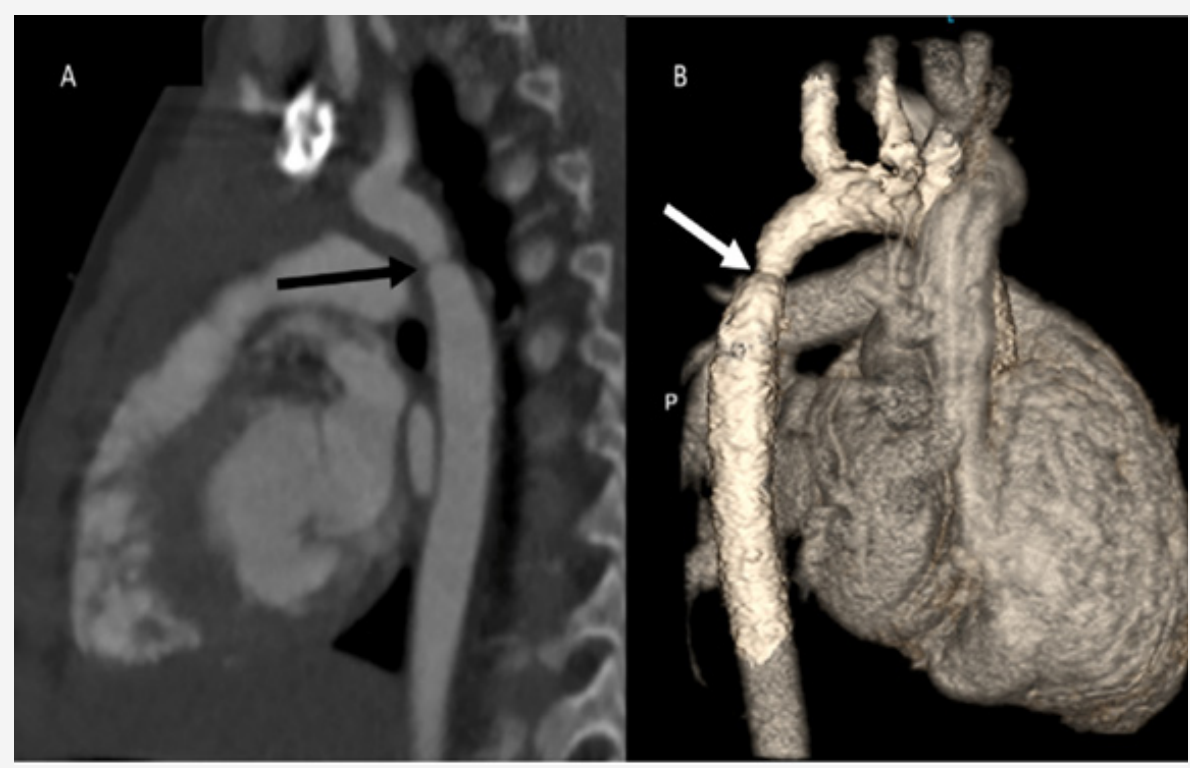

Figure 2: Two (A) and three (B) dimensional Computed tomography of CoA [9].

\section{Magnetic resonance imaging (MRI)}

Cardiac MRI is the optimal initial non-invasive imaging technique that can provide a high-resolution image [13].

\section{Electrocardiogram (ECG)}

Electrocardiogram is used to determine the cardiac rhythm evaluation, ventricular wall hypertrophy and cardiac enlargement.

\section{Chest X-ray}

Chest X-ray may indicate cardiac enlargement, pericardial and pleural effusion and pulmonary congestion.

\section{Surgery}

The intervention either by surgery or cardiac catheterization with stenting of the narrowed segment of aorta is indicated in neonates and infants with severe hypertension, congestive heart failure or systolic blood pressure gradient across the coarctation of aorta more than $20 \mathrm{mmHg}$. Many surgical approaches have been described for correction CoA, but resection of the constricted segment with end to end anastomosis became the standard approach. Cardiac catheterization with balloon angioplasty of the narrowed aortic segment may be used as a substitute for surgical correction, but because of the high incidence of recurrence in the neonates and infants, most cardic surgical centers are shifted toward the surgical correction as the first line in the treatment of CoA in neonates and infants [14].

\section{Preoperative Assessment and Preparation for Surgery}

The anesthetist should perform a thorough review of the history, clinical presentation, current medications, and the patient available investigations. Neonates and infants with hemodynamic instability or cardiogenic shock may benefit from prostaglandin E1 infusion that can be administered via peripheral cannula (or interosseous line) to maintain ductus arteriosus patency. Insertion of arterial and central venous catheters facilitate monitoring the arterial blood pressure and hemodynamic stabilization. Inotropic drugs such as dobutamine, dopamine and epinephrine are indicated to maintain and stabilize the patient hemodynamics. The improvement in patient hemodynamics allows enough time for further imaging techniques such as TTE, CT, and cardiac MRI $[15,16]$. Prostaglandin E1 infusion may cause apnea in neonates, so the patient must be carefully monitored with possibility of the need for intubation and mechanical ventilation. Laboratory work often includes a complete blood count, serum electrolytes, serial arterial blood gases and serum lactate. Cross matched blood must be available before surgery.

\section{Intraoperative Monitoring}

In addition to the standard monitoring described by American Society of Anesthesiologists, preductal invasive arterial pressure monitoring via an arterial catheter placed in the right arm is essential. The left arm shouldn't be used for pressure monitoring because left subclavian artery and descending thoracic aorta are completely occluded by aortic cross clamp to allow resection of the constricted segment and end to end anastomosis [17]. An arterial catheter is usually inserted in the femoral artery to monitor any residual pressure gradient between the upper and lower limbs, although this can be achieved using non-invasive pressure monitoring in most children [18].

The use of bi hemispheric cerebral near-infrared spectroscopy (NIRS) is recommended to allow early detection of cerebral hypoperfusion and ischemia that may result from inappropriate position of aortic clamp or acute heart failure that results in low cardiac output $[19,20]$.

Renal NIRS may be used for monitoring the lower body perfusion during application of aortic cross clamp, but it's not used as a guide for further management [21,22]. A urinary catheter must 
be inserted to monitor urine output. Central venous catheter is often inserted as an access for drug infusion a rarely for monitoring purposes.

\section{Induction and Maintenance of Anesthesia}

The hemodynamic goals on anesthetizing neonates and infants undergoing surgical repair of CoA include maintenance of myocardial contractility, heart rate, preload and avoidance excessive increase or decrease in afterload.

In neonates who are duct dependent, prostaglandins E1 infusion (0.01-0.1 $\mu \mathrm{g} / \mathrm{kg} / \mathrm{min}$ ) should be continued until the removal of aortic cross clamp. Induction of anesthesia must be slow and smooth with titration of low concentration of volatile anesthetic, very low doses of opioid as it may cause profound hypotension in critically ill patients, and muscle relaxants. Single lumen endotracheal tubes provide adequate exposure in neonates and infants.

After endotracheal intubation, arterial and venous catheter placement and urinary catheter insertion, the patient is positioned in the right lateral decubitus position with padding pressure. Hyperthermia $\left(>38^{\circ} \mathrm{C}\right)$ should be prevented to avoid the increased risk of spinal cord injury and paraplegia, passive cooling of the patients to a temperature of $35^{\circ} \mathrm{C}$ is often recommended in an attempt to reduce the risk of neurological injury [23].

\section{The Effects of Aortic Cross Clampingand Declamping}

Heparin 100-150 i $/ \mathrm{kg}$ is administered through a central venous catheter aiming to increase the activated clotting time to 250-300 seconds before application of the clamp. Aortic cross clamping produces an acute additional increase in cardiac afterload. If the left ventricular function is still preserved, the arterial blood pressure proximal to the clamp will markedly increase with concomitant drop in the blood pressure distal to the camp. Proximal hypertension should be maintained to improve the reduced perfusion distal to the aortic clamp because the perfusion of lower body and spinal cord becomes dependent on the collateral circulation and the high proximal arterial pressure [24]. The acute severe increase in proximal blood pressure must be avoided as it may cause acute heart failure and may increase the risk of cerebral stroke. The elevated proximal blood pressure can be controlled by increasing the concentration of volatile anesthetics. Intravenous vasodilators such as sodium nitroprusside, esmolol and nicardipine may be used to control proximal hypertension; however, the use of nitroprusside may be associated with decreased spinal cord perfusion because of steal of blood flow away from spinal cord $[17,19,24]$.

The risk of spinal cord injury can be reduced using cardiopulmonary bypass. Many drugs such as naloxone and steroids have been shown to reduce the risk of spinal cord injury in animal models, but these have not been studied during surgical repair of coarctation of aorta and are not routinely used [24]. Minimizing the aortic cross clamp time to less than (20-30) minutes is well tolerated with reduced risk of spinal cord ischemia [25].

Inotropic drug support (dobutamine, dopamine and epinephrine) is usually required in critically ill patient with failing ventricle. The risk of renal dysfunction can be reduced by minimizing the aortic clamp time and the administration of mannitol 15 minutes before application the aortic clamp.

Removal of aortic clamp is usually associated with hypotension which may be severe, and this can be avoided by discontinuation of vasodilators, lightening the anesthesia, volume expansion and vasopressors [17]. This period of hypotension is usually transient and will be followed by hypertension.

The patient is gradually rewarmed until normothermia. Most patients can be extubated early in the operating room. Critically ill neonates and infants with preoperative heart failure, pulmonary congestion or edema, or anuria should be transferred to intensive care unit (ICU) while they are intubated and mechanically ventilated. These patients are extubated in ICU after hemodynamic stabilization and correction pulmonary, and renal dysfunction.

\section{Postoperative Management}

Postoperative hypertension persists for several weeks after surgical repair of CoA. The serum levels of norepinephrine are increased up to $750 \%$ of preoperative value after CoA repair due to adaptation of baroreceptor)36 (. Increased plasma renin activity may be a contributing factor [26]. Severe postoperative hypertension should be treated with intravenous vasodilators and then replaced by oral agents as early as possible.

Good control of postoperative pain is mandatory to prevent exacerbation of postoperative hypertension. Multimodal analgesia that includes opioids, paracetamol, and nonsteroidal antiinflammatory drugs is the classic management of postoperative pain. Thoracic epidural and paravertebral block have been shown to be effective for pain control after thoracotomy [17,27]. Erector spinae plane block has been used to control post thoracotomy pain with satisfactory results $[28,29]$.

\section{Conclusion}

The hemodynamic goals on anesthetizing neonates and infants undergoing surgical repair of CoA include maintenance of myocardial contractility, heart rate, preload and avoidance excessive increase or decrease in afterload. Clamping the aorta aggravates the proximal hypertension with increased risk of spinal cord injury, myocardial ischemia and renal dysfunction while removal of the aortic clamp is associated with hypotension which may be severe. The risk of spinal cord injury can be reduced by minimizing the time of aortic cross clamp, mild passive hypothermia and the use of cardiopulmonary bypass. Critically ill patients with preoperative heart failure, pulmonary edema or chest infection are not extubated in operating room. 


\section{Acknowledgement}

None.

\section{Conflict of Interest}

No conflict of interest.

\section{References}

1. Joshi G, Skinner G, Shebani SO (2017) Presentation of coarctation of the aorta in the neonates and the infant with short and long term implications. Paediatrics and Child Health 27(2): 83-89.

2. Singh S, Hakim FA, Sharma A, Roy RR, Panse PM, et al. (2015) Hypoplasia, pseudocoarctation and coarctation of the aorta- a systematic review. Heart Lung Circ 24:110-118.

3. Nguyen L, Cook SC (2015) Coarctation of the aorta: strategies for improving outcomes. Cardiol Clin 33: 521-530.

4. Roos Hesselink JW, Scholzel BE, Heijdra RJ, Spitaels SC, Meijboom FJ, et al. (2003) Aortic valve and aortic arch pathology after coarctation repair Heart 89: 1074-1077.

5. Engel MS, Kochilas LK (2016) Pulse oximetry screening: a review of diagnosing critical congenital heart disease in newborns. Med Devices (Auckl) 9: 199-203.

6. Franklin O, Burch M, Manning N, Sleeman K, Gould S, et al. (2002) Prenatal diagnosis of coarctation of the aorta improves survival and reduces morbidity. Heart 87: 67-69.

7. Familiari A, Morlando M, Khalil A, Sonesson SE, Scala C, et al. (2017) Risk factors for coarctation of the aorta on prenatal ultrasound: a systematic review and meta-analysis. Circulation 135: 772-785.

8. Goudar SP, Shah SS, Shirali GS (2016) Echocardiography of coarctation of the aorta, aortic arch hypoplasia, and arch interruption: strategies for evaluation of the aortic arch. Cardiol Young 26: 1553-1562.

9. Doshi AR, Chikkabyrappa S (2018) Coarctation of Aorta in Children Cureus 10(12): e3690.

10. Dijkema EJ, Leiner T, Grotenhuis HB (2017) Diagnosis, imaging and clinical management of aortic coarctation. Heart 103: 1148-1155.

11. Nie P, Wang X, Cheng Z, Duan Y, Ji X, et al. (2012) The value of low-dose prospective ECG-gated dual-source CT angiography in the diagnosis of coarctation of the aorta in infants and children. Clin Radiol 67: 738-745.

12. Gach P, Dabadie A, Sorensen C, Quarello E, Bonello B, et al. (2016) Multimodality imaging of aortic coarctation: from the fetus to the adolescent. Diagn Interv Imaging 97: 581-590.

13. Shepherd B, Abbas A, McParland P, Fitzsimmons S, Shambrook J, et al. (2015) MRI in adult patients with aortic coarctation: diagnosis and follow-up. Clin Radiol 70: 433-445.

14. Rao PS (2020) Neonatal (and Infant) Coarctation of the Aorta Management Challenges Research and Reports in Neonatology 10: 11 22.

15. Liberman L, Gersony WM, Flynn PA, Lamberti JJ, Cooper RS, et al (2004) Effectiveness of prostaglandin E1 in relieving obstruction in coarctation of the aorta without opening the ductus arteriosus. Pediatr Cardiol 25: 49-52.

16. Kouchoukos NT, Blackstone EH, Hanley FL, Kirklin JK (2012) Coarctation of the aorta and interrupted aortic arch. In: Kirklin/Barratt-Boyes Cardiac Surgery $4^{\text {th }}$ (edn.), Elsevier Health Sciences, Netherlands, pp. $1717-1779$.

17. Spaeth JP, Loepke AW (2015) Anesthesia for left-sided obstructive lesions. In: Andropoulos DB, Stayer SA, Mossad EB, Miller Hance WC (eds.) Anesthesia for Congenital Heart Disease $3^{\text {rd }}$ (edn.), Wiley Blackwell, USA, pp. 497-515.

18. Maddali MM, Valliattu J, Delamie T, Zacharias S (2008) Selection of monitoring site and outcome after neonatal coarctation repair. Asian Cardiovasc Thorac Ann 16: 236-239.

19. Moerman A, Bové T, François K, Jacobs S, Deblaere I, et al. (2013) The effect of blood pressure regulation during aortic coarctation repair on brain, kidney, and muscle oxygen saturation measured by near-infrared spectroscopy: a randomized, clinical trial. Anesth Analg 116: 760-766.

20. Berens RJ, Stuth EA, Robertson FA, Jaquiss RD, Hoffman GM, et al. (2006) Near infrared spectroscopy monitoring during pediatric aortic coarctation repair. Paediatr Anaesth 16: 777-781.

21. Kim HJ, Park JH (2011) Cerebral and somatic oxygenation monitoring during pediatric aortic coarctation repair using near infrared spectroscopy: 10AP3-7. Eur J Anaesthesiol 28: 154.

22. Fuentes García D, Cárceles Barón MD, López López R, Roqués Escolar V (2010) Non-invasive oximetry for early detection of cerebral and somatic ischaemia during corrective surgery for aortic coarctation in paediatric patients. Br J Anaesth 104: 780-782.

23. Crawford JF, Sade R (1984) Spinal cord injury associated with hyperthermia during aortic coarctation repair. J Thorac Cardiovasc Surg 87: 616- 618.

24. Griepp RB, Griepp EB (2007) Spinal cord perfusion and protection during descending thoracic and thoracoabdominal aortic surgery: the collateral network concept. Ann Thorac Surg 83: S865-S869.

25. Krieger KH, Spencer FC (1985) Is paraplegia after repair of coarctation of the aorta due principally to distal hypotension during aortic crossclamping? Surgery 97: 2-7.

26. Rocchini AP, Rosenthal A, Barger AC, Castaneda AR, Nadas AS (1976) Pathogenesis of paradoxical hypertension after coarctation resection. Circulation 54: 382-387.

27. Türköz A, Balci ST, Can Güner M, Ulugöl H, Vuran C, et al. (2013) Anesthesia management with single injection paravertebral block for aorta coarctation in infant. Paediatr Anaesth 23: 1078-1083.

28. Tulgar S, Selvi O, Ozer Z (2018) Clinical experience of ultrasound-guided single and bi-level erector spinae plane block for postoperative analgesia in patients undergoing thoracotomy. J Clin Anesth 50: 22-23.

29. Kelava M, Anthony D, Elsharkawy H (2018) Continuous erector spinae block for postoperative analgesia after thoracotomy in a lung transplant recipient. J Cardiothorac Vasc Anesth 32: e9-e11. 